\title{
Design of a Symmetrical Quad-rotor Biplane Tail-Sitter Aircraft without Control Surfaces and Experimental Verification
}

\author{
Hongyu Wang ${ }^{1}$, Xun Zhao ${ }^{1}$, Hui Bai ${ }^{2}$,Cunyue Lu ${ }^{1}$, Baomin Zhang ${ }^{1}$,Cong Li \\ ${ }^{1}$ Department of Instrument Science and Engineering, Shanghai Jiao Tong University, Shanghai, China \\ ${ }^{2}$ School of Information Science \& Technology, Donghua University, Shanghai, China
}

\begin{abstract}
This paper presents the design of a symmetrical quad-rotor biplane tail-sitter VTOL UAV (Vertical Takeoff and Landing Unmanned Aerial Vehicle) which is composed of four rotors and two symmetrically mounted fixed wings. This aircraft achieves high accuracy in the attitude control and smooth flight mode transition with four rotors rather than the conventional VTOL UAVs using control surfaces. The proposal of angled rotor mounting is adopted to address the issue of insufficient yaw control authority. The layout of symmetrically mounted fixed wings makes the aircraft have capability of rapid bidirectional flight mode transition to improve maneuverability. To validate the performance of the aircraft, simulation and flight experiments are both implemented. These results show that the aircraft has a rapid yaw response under condition of the stable attitude control. In comparative experiment, it is shown that the aircraft is more flexible than other similar configuration of aircrafts. This symmetrical quad-rotor biplane tailsitter VTOL UAV will have a wide range of potential applications in the military and civilian areas due to its superior performance..
\end{abstract}

\section{Introduction}

In recent years, the potential uses for UAVs have increased drastically. These applications include but not limited to surveillance, rescue and reconnaissance, as well as medical supply, consumer package delivery and power line inspection [1]. The VTOL aircrafts can either take off and land vertically or fly in a high-speed cruise configuration [2]. At present, according to different ways to achieve flight mode transition, VTOL aircrafts may be categorized as tilt-rotor, tilt-wing, tail-sitter aircrafts, et al [3-6]. The simplest way is tail-sitting since it switches between level flight and hover flight mode by changing its pitch angle of the fuselage [7]. Tail-sitter aircrafts have been extensively studied for its simple and effective flight mode transition. Stone studied the T-Wing, which has a canard wing and tandem rotors [8]. Kita developed a simple tail-sitter VTOL UAV with a single propeller $\mathrm{R} / \mathrm{C}$ airplane [9]. These tail-sitter aircrafts which are developed based on fixed-wing aircrafts, have an accuracy drawback in the attitude control for stationary hovering because of the use of control surfaces, such as aileron, elevator, and rudder. To overcome such a drawback, those tail-sitter aircrafts which are developed based on rotorcrafts have been a research hotspot. This strategy was proposed by Young at NASA Ames Research Centre in 2002 [10]. Oosedo designed a quadrotor tail-sitter aircraft with one wing and succeeded in manual flight in 2013 [11, 12]. Bogdanowicz developed a quad-rotor biplane micro air vehicle in 2015 [13]. The cambered wing makes those aircrafts have different aerodynamic efficiency when they achieve bidirectional flight mode transition, which causes that they are inflexible. To address these issues above, a new symmetrical quad-rotor biplane configuration tail-sitter VTOL UAV is proposed. This aircraft realizes high accuracy in attitude control and smooth flight mode transition with four rotors. To solve the slow response of yaw and the insufficient yaw control authority problems, the proposal of angled rotor mounting is adopted. The configuration of symmetrical biplane enables the aircraft to have the same aerodynamic efficiency during bidirectional flight mode transition, which is a compromise between aerodynamic efficiency and maneuverability.

In order to verify the rationality of the design, the mathematical model is established and control system is designed. The flight experiments are carried out to evaluate the simulation results. A comparative experiment with quad-rotor monoplane and asymmetrical quad-rotor biplane is also implemented to evaluate the maneuverability of the aircraft.

\section{Principle of flight}

The symmetrical quad-rotor biplane tail-sitter VTOL UAV without Control surfaces has three flight modes: hovering mode, transitional flight mode and level flight mode, as illustrated in Fig. 1. In hovering mode, the 
force generated by rotors is mainly used to maintain the gravity. The aircraft has accurate attitude control with excess thrust in hovering mode. The transition between flight modes is attained through a pitching moment provided through RPM variation of the propellers. Varying RPM of propeller pairs allows for differential thrust which, when coupled with the installed moment arm, generates a sizeable pitching moment. Transition flight mode is between hovering mode and level flight mode. The configuration of symmetrical biplane makes the aircraft has the same aerodynamic efficiency to achieve a rapid bidirectional transition. It can be along with either the A path or the B path for transition, which improves the maneuverability of the aircraft, as illustrated in Fig.. 1. As the aircraft enters the level flight mode, at sufficiently high cruise speeds, the loads are then transferred to the wing. So, the force generated by rotors is mainly used to increase the cruise speed.

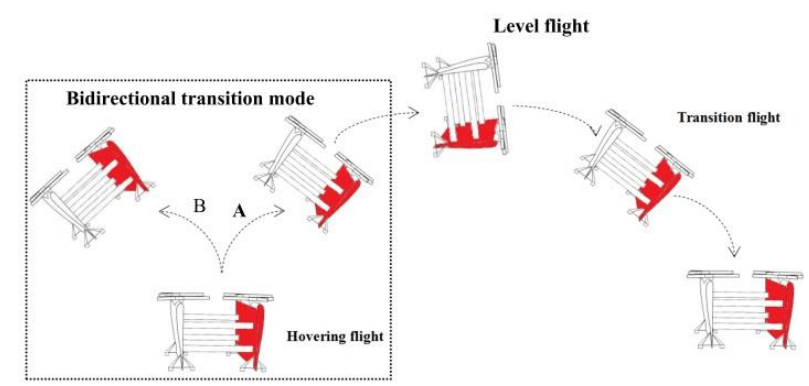

Figure 1. Concept of flight mode.

Table 1. Nomenclature

\begin{tabular}{|c|c|c|}
\hline Symbol & Unit & Description \\
\hline$i$ & & Index of each motor \\
\hline$j$ & & Index of each axis \\
\hline$\Omega_{i}$ & $(\mathrm{rpm})$ & The $i^{\text {th }}$ motor speed \\
\hline$l$ & $(m)$ & $\begin{array}{l}\text { Distance of perpendicular } \\
\text { form each motor to the center }\end{array}$ \\
\hline$C_{T}$ & & $\begin{array}{l}\text { Torque coefficient of each } \\
\text { propeller }\end{array}$ \\
\hline$C_{M}$ & & $\begin{array}{l}\text { Lift coefficient of each } \\
\text { propeller }\end{array}$ \\
\hline$a$ & $(\mathrm{rad})$ & Tilt angle of each motor \\
\hline$\alpha$ & $(\mathrm{rad})$ & Angle of attack \\
\hline$m$ & $(k g)$ & Mass of whole aircraft \\
\hline$g$ & $\left(m / s^{2}\right)$ & Gravitational acceleration \\
\hline$\tau_{j}$ & $(N / m)$ & Torque of each axis \\
\hline$p$ & $(\mathrm{rad} / \mathrm{s})$ & Angular velocities of $\mathrm{X}$ axis \\
\hline$q$ & $(\mathrm{rad} / \mathrm{s})$ & Angular velocities of $Y$ axis \\
\hline$r$ & $(\mathrm{rad} / \mathrm{s})$ & Angular velocities of $Z$ axis \\
\hline$u$ & $(m / s)$ & $\begin{array}{l}\text { Translational velocities of } \mathrm{X} \\
\text { axis }\end{array}$ \\
\hline$v$ & $(m / s)$ & $\begin{array}{l}\text { Translational velocities of } \mathrm{Y} \\
\text { axis }\end{array}$ \\
\hline$w$ & $(m / s)$ & $\begin{array}{l}\text { Translational velocities of } \mathrm{Z} \\
\text { axis }\end{array}$ \\
\hline$I_{j}$ & $\left(k g * m^{2}\right)$ & $\begin{array}{l}\text { Fuselage moment of inertia of } \\
\text { each axis }\end{array}$ \\
\hline
\end{tabular}

\begin{tabular}{|c|c|l|}
\hline$\theta$ & $(\mathrm{rad})$ & Pitch angle \\
\hline$\phi$ & $(\mathrm{rad})$ & Roll angle \\
\hline$\psi$ & $(\mathrm{rad})$ & Yaw angle \\
\hline$L$ & $(\mathrm{~N})$ & Lift of wings \\
\hline$D$ & $(\mathrm{~N})$ & Drag of wings \\
\hline$Q_{\text {slipstream }}$ & $(\mathrm{N} / \mathrm{m})$ & Torque of propeller slipstream \\
\hline$J_{r}$ & & $\begin{array}{l}\text { propeller gyroscopic effect } \\
\text { coefficient }\end{array}$ \\
\hline$M_{w}$ & $(\mathrm{~N} / \mathrm{m})$ & $\begin{array}{l}\text { Pitching moment of the } \\
\text { longitudinal area }\end{array}$ \\
\hline$M_{\text {lat }}$ & $(\mathrm{N} / \mathrm{m})$ & $\begin{array}{l}\text { Pitching inertial resistance of } \\
\text { the lateral area }\end{array}$ \\
\hline
\end{tabular}

\section{Configuration}

\subsection{Fuselage}

An overview the aircraft is shown in Fig. 2. It consists of two pairs of counter rotating propellers arranged in a quad-rotor configuration. Two wings are affixed symmetrically to each pair of rotors through a set of rigid motor attachments. The wings are constructed from low density polyurethane foam using high lift, low Reynolds number airfoil. The battery and on-board microcontroller are incorporated in the centre of the vehicle. Each rotor shaft is tilted inward at an angle toward the mid-span of its respective wing.

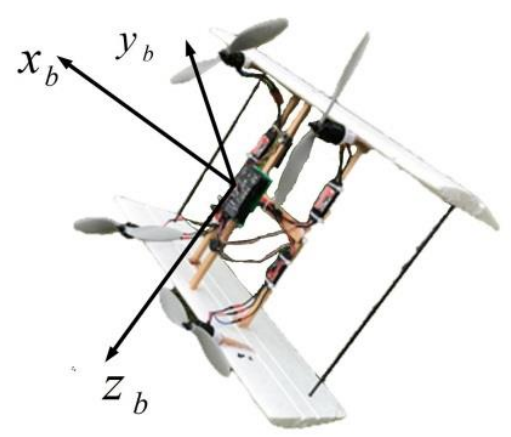

Figure 2. Overview of the aircraft.

\subsection{Angled rotor mounts}

The aircraft suffers from the insufficient yaw control authority, especially, when it enters the level flight mode, which results in the divergence of the yaw. The proposal of angled rotor mounting is adopted to address the issue, as show in Fig. 3. Each rotor shaft is tilted inward at an angle a, toward the mid-span of its respective wing. $F_{z}$ is the force generated by rotor. $F_{V}$ is the vertical component of $F_{z} \cdot F_{H}$ is the horizontal component of $F_{z}$. These sideforces will provide a valuable menment which will compensate for yaw to attained sufficient yaw control authority, though, the vertical commopent of the force generated by rotor is decreased. Fig. 4 shows the direction and relative magnitude of the new forces which, 
when coupled with a moment arm, will generate a large yaw moment in the clockwise direction, which is the desired direction of rotation. The new yaw moment is given by Eq.1.

$$
\tau_{z}=\left(C_{M}+\sqrt{2} C_{T} l \sin a\right)\left(\Omega_{1}^{2}-\Omega_{2}^{2}+\Omega_{3}^{2}-\Omega_{4}^{2}\right)
$$

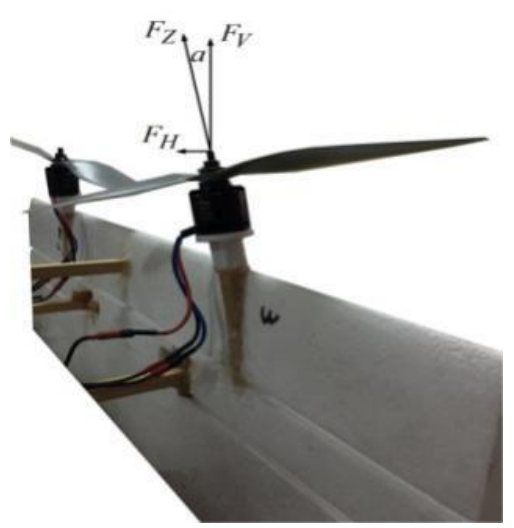

Figure 3. Diagram of thrust.

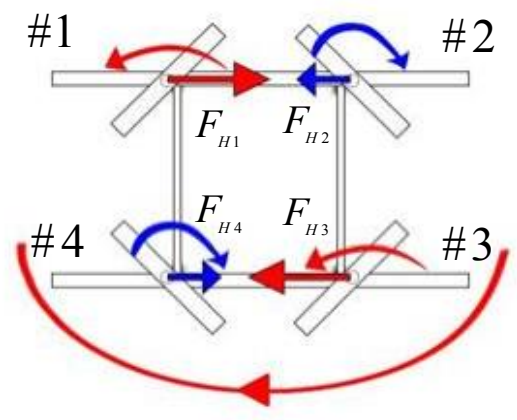

Figure 4. Diagram of thrust vectoring.

\section{Modelling}

\subsection{Coordinate systems}

The aircraft body coordinates $\left(X_{b}, Y_{b}, Z_{b}\right)$ are defined as shown in Fig. 2. The earth-fixed coordinates define the $X_{i}$-axis as true north, the $Y_{i}$-axis as east and $Z_{i}$-axis as perpendicular downward. The $X_{b}$-axis of the aircraft coordinates coincides with the $X_{i}$-axis of the inertial coordinates when pitch and yaw angles are equal to 0 .

\subsection{Dynamics}

The equations of translational and rotation motion of the aircraft are described by Eq.2-Eq.7. The effect of the slipstream on the wing is modelled as a yaw moment.

$$
\begin{aligned}
& \dot{u}=\frac{r v-q w-L \cos \alpha-D \sin \alpha}{m}+g \cos \theta \cos \psi \\
& \dot{v}=\frac{p w-r u}{m}+g \cos \theta \sin \psi \\
& \dot{w}=\frac{q u-p v-L \sin \alpha-D \cos \alpha-T}{m}+g \sin \theta \\
& \dot{p}=\frac{\left(I_{y}-I_{z}\right)+\tau_{x}-J_{r} r \sum_{i=1}^{4}(-1)^{i} \Omega_{i}}{I_{x}} \\
& \dot{q}=\frac{\left(I_{z}-I_{x}\right)+\tau_{y}+M_{x}+M_{\text {lat }}-J_{r} p \sum_{i=1}^{4}(-1)^{i} \Omega_{i}}{I_{y}} \\
& \dot{r}=\frac{\left(I_{x}-I_{y}\right)+\tau_{z}+Q_{\text {slipstream }}}{I_{z}}
\end{aligned}
$$

\section{Control systems}

\subsection{Attitude and Altitude Control System}

The attitude and altitude controller is designed based on cascaded PID controller, as shown in Fig. 5. The cascaded PID controller can improve the dynamic performance and robustness of the system. In order to address the issue of excessive overshot, which is caused by the frequent change of the reference angular velocity, the inner loop adopts differential forward PID controller. As shown in Fig. 6, the differential PID controller only differentiates the feedback signals rather than the reference signals. As shown in Fig. 5, firstly, the reference and current attitude are transformed into rotation matrices in avoidance of the "gimbal lock". Those rotation matrices are sent to the outer PID controller to get the reference angular velocity. Then, reference angular velocity, current angular velocity, reference altitude and current altitude are sent to the differential forward PID to get desired force and moments. Finally, the force and these moments are assigned to each rotor to achieve the attitude and altitude control.

\subsection{Transition Control System}

An important difference in aircraft dynamics between hovering and level flight mode is the lift generated by the wings. Transition flight, which refers to the flight phase between level flight and hovering, is supported by both thrust and lift. The aircraft is aerodynamically unstable during transition flight because a stall occurs at the fixed wings. The biggest problem is the change of the altitude. It is hard for a pilot to operate to get an optimal thrust at different speed and attack angel. So the transition control system is designed for constant altitude flight to attain the automatic transition, as shown in Fig. 7. The optimization problem of this transition strategy is solved by Levenberg-Marquardt algorithm. Under condition of constant altitude, optimal thrust will be obtained at every velocity and attack angel using Levenberg-Marquardt algorithm. For flight safety, the transition mode switch is set in the RC transmitter to ensure that the pilot has sufficient authority for the aircraft. 


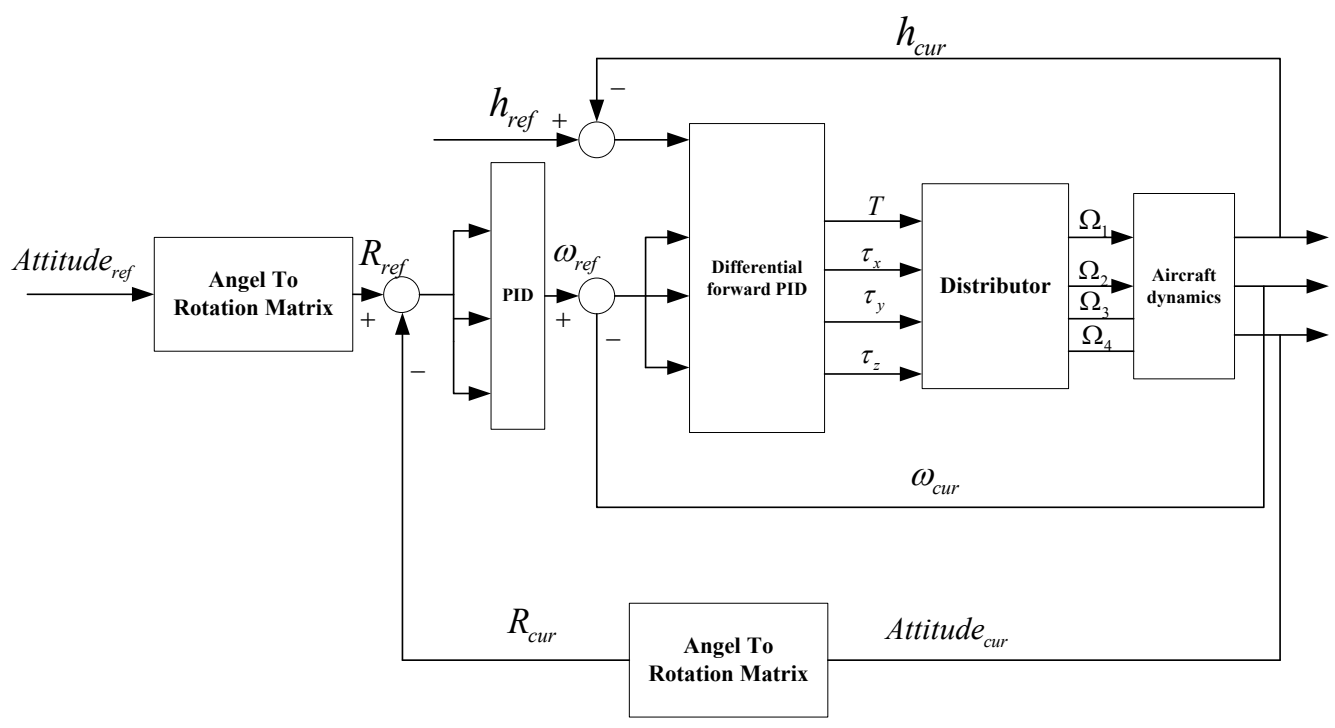

Figure 5. Attitude and altitude control system.

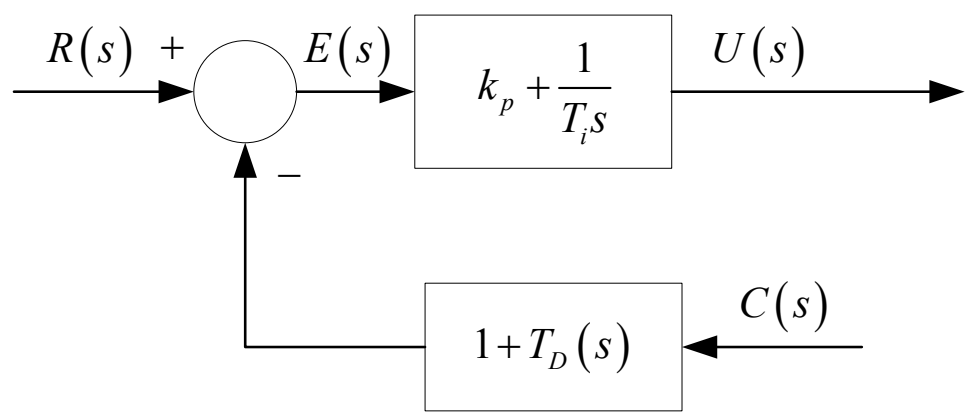

Figure 6.Differential forward PID controller.

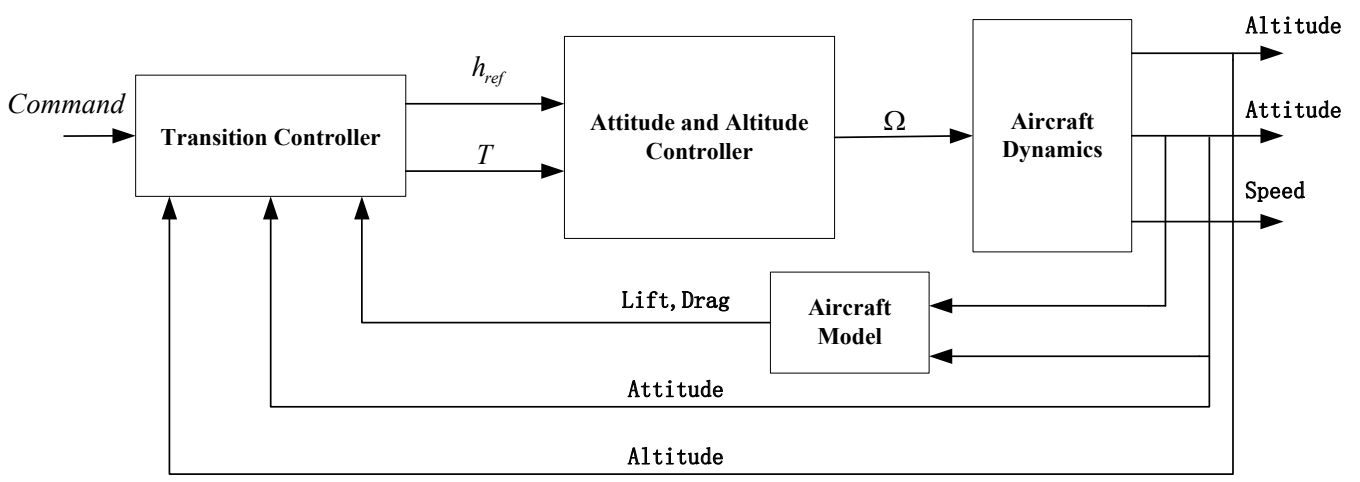

Figure 7. Transition control system.

\section{Simulation and flight experiment}

\subsection{Simulation}

The simulation experiments are composed of the aircraft taking off, the hovering mode and the transition mode at time of 5s. The simulation results are shown in Fig. 8. As can be seen in Fig. 8(a), the aircraft has rapid response under condition of high accuracy in the attitude control during either the hovering mode or the transition mode. The force generated by rotors is used to balance the gravity in hovering mode. At the beginning of transition mode, the force generated by wings is small due to small translational velocity while the vertical component of the force generated by rotors is still decreasing. For maintaining the altitude, the force generated by rotors needs to be increased. The lift generated by wings which is mainly used to maintain the gravity of the aircraft 
increases as the velocity increases. So, the force for lift which generated by rotors is continuously reduced, as shown in Fig. 8(b). The force generated by rotors is mainly used to increase the speed of the aircraft, as shown in Fig. 8(c). The cruise speed reaches up to $13 \mathrm{~m} / \mathrm{s}$ while the max speed of a quad-rotor aircraft is about $8 \mathrm{~m} / \mathrm{s}$. Fig. 8(d) shows that the yaw is divergent before rotors tilting, especially during transition mode because of insufficient yaw control authority and influences of streamslip. The results that the yaw is convergent after rotors tilting validate that the proposal of angled rotor mounting is effective.
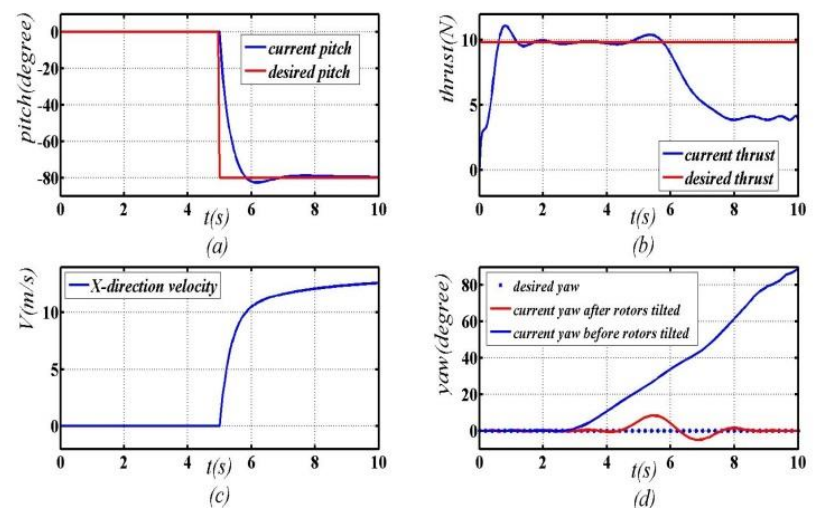

Figure 8. Results of simulation.

\subsection{Flight experiment}

The flight experiments are composed of evaluating the simulation results and comparative experiment with other configuration aircrafts. The results of the flight experiment as shown in Fig. 9. As can be seen in these figures, the results of flight experiment and simulation are basically consistent, except that the altitude change in flight experiment is more severe than it in simulation during transition flight mode. The reason is that the wind is not taken into consideration when the transition controller is designed. The comparative experiment is that a round-trip flight with a straight-line distance of $100 \mathrm{~m}$ is carried out with three different configuration aircrafts. These aircrafts are the same except for the configuration. The results of comparative experiment with other aircrafts are listed in Table 2. As can be seen in Table 2, the configuration which is proposed by this paper has the minimal transition time and the minimal total time. So, the results of comparative experiment validate that the layout of symmetrical biplane improves the maneuverability of the aircraft.

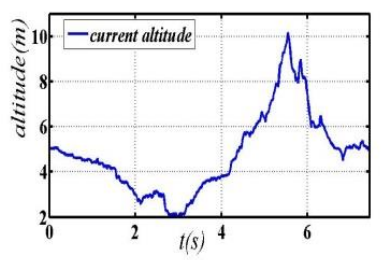

(a)

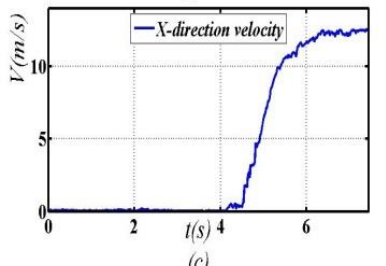

(c)

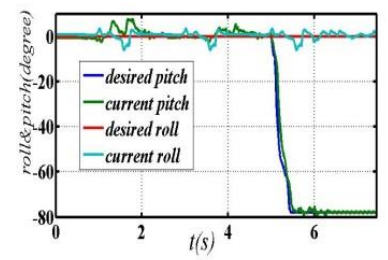

(b)

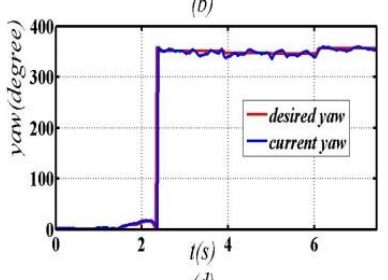

$(d)$

Figure 9. Results of flight experiment.

Table 2. Results of comparison experiment

\begin{tabular}{|l|c|c|c|}
\hline & & & \\
\hline Configuration & Symmetrical biplane & Asymmetric biplane & Monoplane \\
\hline $\begin{array}{l}\text { Max speed after forward X- } \\
\text { direction transition }\end{array}$ & $12.5(\mathrm{~m} / \mathrm{s})$ & $13(\mathrm{~m} / \mathrm{s})$ & $14(\mathrm{~m} / \mathrm{s})$ \\
\hline $\begin{array}{l}\text { Max speed after negative X- } \\
\text { direction transition }\end{array}$ & $12.5(\mathrm{~m} / \mathrm{s})$ & $8(\mathrm{~m} / \mathrm{s})$ & $9(\mathrm{~m} / \mathrm{s})$ \\
\hline Time of mode transition & $4.5(\mathrm{~s})$ & $4.2(\mathrm{~s})$ & $3.88(\mathrm{~s})$ \\
\hline Time of AB experiment & $14.5(\mathrm{~s})$ & $17.8(\mathrm{~s})$ & $16.0(\mathrm{~s})$ \\
\hline
\end{tabular}

\section{Summary}

In this work, a symmetrical quad-rotor biplane tail-sitter VTOL UAV without control surfaces is designed and mathematic model is established which is used for designing control system. Simulation and flight experiments are also carried out. The results of experiment verify the aircraft can achieve high accuracy in attitude control during hovering mode. The proposal of angled rotor mounting speeds up the response of yaw and increase the yaw control authority by compensating the moment of yaw. The results of the comparative flight experiment comfirm that the layout of symmetrical biplane improves the maneuverability of the aircraft. This symmetrical quad-rotor biplane tail-sitter VTOL UAV will have a wide range applications in the military and 
civilian areas due to it's superior performance. In the future, the work on how to enhance the robustness of the transition control system and how to realize hovering precisely in strong wind conditions will be carried out.

\section{References}

1. K.P.Valavanis, Andvances in Unmanned Aerial Vehicle:State of Art and the Road to Autonomy(2007)

2. Abdessameud A, Tayebi A, Automatica J.IFAC, 47, 2383-2394(2011)

3. Green W E, OhPY, ICRA, 2164-2169(2006)

4. Maisel, Martin D., D. J. Giulianetti, D. C. Dugan. The History of the XV-15 Tilt Rotor Research Aircraft from Concept to Flight (2000)
5. Taylor, Frederick.M, V/stol aircraft with variable tilt wing(US, US 3666209 A, 1972)

6. Kubo D, Suzuki S, J.AIRCRAFT, 45.1, 292297(2008)

7. Stone R H, Anderson P, Hutchison C, J.AIRCRAFT, 45.2, 673-685(2008)

8. Kita K, Konno A, Uchiyama M, IJRM,21.2,77(2009)

9. Young L, Aiken E, Johnson J, AIAA(2002)

10. Oosedo A, Abiko S, Konno A, ICRA, 317-322(2013)

11. Oosedo A, Abiko S, Konno A, Autonomous Robots, 1-17(2006)

12. Hrishikeshavan V, Bawek D, Rand O, ISMUR(2013)

13. Bogdanowicz C M, J.AIAA(2015) 